\title{
Factores que influyen en la decisión de estudiar ingeniería comercial según la perspectiva del alumnado de los sistemas público y privado de la Región de Coquimbo, Chile
}

\author{
Sebastián C. Araya-Pizarro(1)* y Camilo R. Araya-Pizarro(2) \\ (1) Facultad de Cs. Sociales y Económicas, Dpto. de Cs. Económicas y Empresariales, Universidad de La Serena, \\ Amunátegui s/n, La Serena-Chile. (correo-e: saraya@userena.cl) \\ (2) Facultad de Economía y Negocios, Universidad Santo Tomás, Ruta 5 Norte s/n, La Serena-Chile \\ (correo-e: carayap@santotomas.cl)
}

* Autor a quien debe ser dirigida la correspondencia

Recibido Jul. 24, 2019; Aceptado Sep. 24, 2019; Versión final Nov. 8, 2019, Publicado Abr. 2020

\begin{abstract}
Resumen
La investigación contrasta los factores que influyen en la decisión de los jóvenes para estudiar ingeniería comercial en base a la comparación de las motivaciones y preferencias del alumnado de los sistemas público y privado de la Región de Coquimbo (Chile). Los datos, recolectados mediante una encuesta realizada a estudiantes de primer año de la carrera, fueron analizados a través de pruebas inferenciales y la aplicación de un modelo multivariado. Los resultados mostraron que los estudiantes del sistema privado basan su elección en la vocación, salida laboral y motivaciones vinculadas al emprendimiento e innovación; mientras que los alumnos del sistema público valoran la salida laboral, crecimiento personal y motivaciones relacionadas con la superación personal y posición económica. El contraste de las preferencias evidenció diferencias en la importancia relativa de atributos tales como acreditación de la carrera y reconocimiento institucional. Se devela que el conocimiento previo de la carrera (empleabilidad e ingresos), constituye un factor clave de su preferencia.
\end{abstract}

\section{Factors influencing the decision to study commercial engineering according to the perspective of the students of the public and private systems of the Coquimbo Region, Chile}

\begin{abstract}
The research compares the factors that influence the decision of young people to study commercial engineering based on the comparison of the motivations and preferences of university students of the public and private systems of the Coquimbo Region (Chile). The data, collected through a survey of freshmen was analyzed through inferential tests and the application of a multivariate model. The results showed that students of the private system base their choice on vocation, career employability level and motivations related to entrepreneurship and innovation; while students of the public system select the career by the career employability level, personal growth and motivations related to personal improvement and economic position. The comparison of the preferences showed differences in the relative importance of the attributes accreditation of the career and institutional recognition. It is revealed that knowledge of the career (employability and income) is a key factor of their preference.
\end{abstract}




\section{INTRODUCCIÓN}

Cuando un estudiante finaliza su enseñanza secundaria debe enfrentar, además de la elección de la carrera a estudiar, una decisión fundamental: ¿en qué universidad hacerlo: pública o privada? Esto conduce a diferentes cuestionamientos sobre las motivaciones y preferencias que resultan determinantes en su selección y que podrían estar asociados al tipo de sistema educativo por el cual se inclinan. El presente estudio planteó por objetivo contrastar las motivaciones y preferencias declaradas por estudiantes universitarios del sistema público y privado respecto a su decisión de estudiar la carrera de mayor matrícula en Chile: ingeniería comercial, un área de la ingeniería administrativa centrada en formar profesionales con competencias en gestión estratégica y negocios.

La revisión de la literatura referida al tópico de investigación muestra que las motivaciones de los estudiantes, constituyen factores determinantes de su elección de carrera (Cheng, 2019; Said-Hung, Gratacós y Valencia, 2017; Sheldon y Corcoran, 2019). Asimismo, es posible denotar que las preferencias de las personas dependen de la valoración (importancia) que asignan a las características que poseen los diferentes programas educacionales que les son ofrecidos, como por ejemplo: arancel anual, planta académica, infraestructura, acreditación, entre otros (Araya-Pizarro et al., 2015). Por tanto, esta investigación pretende descubrir, en base al contraste del sistema educativo al que pertenece el estudiante (público o privado), qué diferencias existen entre los factores motivacionales que impulsan la elección de una carrera y los atributos valorados de esta, todo ello enmarcado, en un contexto diferente a los habitualmente estudiados (América del Norte, Europa y Asia), como lo es caso de los países latinoamericanos.

Se espera que los hallazgos obtenidos proporcionen a las casas de estudios información relevante para que elaboren sus programas educacionales tomando en cuenta, las motivaciones y preferencias de sus estudiantes, contribuyendo así a contrarrestar dos problemas trascendentales del sistema educativo actual: el bajo rendimiento académico y la deserción del estudiantado (Ortiz et al., 2014; Rodriguez et al., 2018). También, los resultados pueden ser de interés para la formulación de políticas públicas en educación superior, ya que el conocimiento empírico de los motivos que llevan al alumnado a elegir una carrera, ayuda a las instituciones de educación superior (públicas y privadas) a mejorar sus currículos, focalizándose en los intereses actuales del estudiantado. Ello favorece la adaptación del educando al sistema universitario, aumenta la calidad de vida universitaria y como consecuencia, impacta, positivamente, en los índices de titulación oportuna, retención estudiantil e inserción sostenible de los titulados en el mercado laboral.

En la actualidad, la elección de carrera es un campo de estudio ampliamente explorado, sobre todo el análisis de las motivaciones y de sus preferencias determinantes (Ashari et al., 2019; Bergmark et al., 2018; Bukuluki et al., 2019; García-Ripa et al., 2018; Karabulut y Erkanli, 2017; Marinas et al., 2016; Ng et al., 2016; Quattrocchi et al., 2017). Históricamente, en el ámbito motivacional, se encuentran diversos marcos contextuales que abordan su concepción (por ejemplo: la teoría de la atribución de Heider, la teoría socialcognitiva de Bandura, la teoría de autorregulación de Kuhl y la teoría de metas de logro de Elliot y Dweck) destacando, como enfoque teórico del presente estudio, la Teoría de la Autodeterminación de Deci y Ryan (2000). Bajo esta perspectiva, se busca explicar la influencia de ciertos procesos subjetivos en la elección de una profesión, atribuyendo las causas tanto a factores intrínsecos como extrínsecos de la motivación (Cheng, 2019; Ho et al., 2018). Es decir, la motivación, entendida como la fuerza que mueve a realizar una actividad o comportamiento, puede nacer de una necesidad que se genera de forma inherente y autónoma o bien puede ser inducida externamente (Stover et al., 2017), donde las preferencias y los intereses personales han demostrado ser motivos claves a la hora de elegir qué carrera estudiar (Rodríguez-Muñiz et al., 2018).

Los estudios realizados en el ámbito de la elección por una carrera son extensos y se pueden encontrar investigaciones aplicadas bajo diferentes metodologías y contextos. Bravo y Vergara (2018), por ejemplo, en un estudio desarrollado en Colombia, revelaron que los intereses personales de los estudiantes son el factor determinante al momento de la elección, tanto en los colegios públicos como en los privados, de forma que prefieren la satisfacción que les produce realizar una tarea que les gusta, en vez de otros factores externos como la generación de beneficios a la comunidad o el salario futuro. Karabulut y Erkanli (2017) exploraron las razones que influyen en la elección de carrera de los estudiantes de Administración en Turquía, observando que se ven afectados principalmente por las opiniones de sus amigos y el entorno social en el que se encuentran. Said-Hung et al. (2017) analizaron, en Colombia, las motivaciones que tienen mayor peso, al momento de elegir carreras relacionadas con la Pedagogía, encontrando que las motivaciones de tipo altruistas e intrínsecas son más valoradas que las motivaciones de tipo extrínsecas. Nyamwange (2016), en Kenia, analizó la influencia del interés en las decisiones de elección de carrera de pregrado en estudiantes universitarios de primer año, confirmando la importancia del conocimiento previo sobre esta. Villamizar y Delgado (2017) analizaron los motivos por estudiar psicología en Colombia, encontrando que los factores de mayor peso están los motivos afiliativos, seguidos por los de logro y prestigio. Marinas et al. (2016) examinaron los factores que indicen en la elección por estudiar programas de administración y negocio en 
Rumania, hallando que las razones de preferencia se basan en aspectos extrínsecos, como las expectativas de empleo, factores interpersonales (en particular la familia) y la exposición temprana a las profesiones. $\mathrm{Ng}$ et al. (2016) exploraron los factores que pueden vincularse con una elección de carrera de postgrado en administración en el sector público y privado en Botswana, encontrando que, a diferencia de otros estudios sobre la motivación en el sector público, los factores intrínsecos y el altruismo no son predictivos en la selección de la carrera. En una línea similar, Diwan et al. (2013) contrastaron las motivaciones y las aspiraciones profesionales de estudiantes indios de medicina, revelando que no existen diferencias significativas entre estudiantes del sector público y privado. Por otro lado, Maniu y Maniu (2014) develaron que los factores determinantes en la elección de un plantel universitario son la reputación institucional, el costo, la salida laboral, la influencia parental, la oferta educativa y la ubicación.

Por último, en Chile, Zuniga-Jara et al. (2019) hallaron que la reputación de la universidad y el acceso a beneficios económicos son los factores más importantes para elegir estudiar ingeniería comercial en la Región de Coquimbo. Alonso-Dos-Santos et al. (2017), por su parte, identificaron, también en estudiantes de ingeniería comercial (Región del Bío-Bío), que el motivo prioritario de elección de la carrera es su gran proyección laboral. Finalmente, Araya-Pizarro et al., (2015), en un estudio realizado sobre las preferencias por un programa de posgrado en la Región de Coquimbo, revelaron como factores determinantes el reconocimiento de la institución y la modalidad de clases (full-time, weekend, online). En conclusión, la revisión teórica demuestra que, independientemente de la carrera o país de estudio, en las decisiones de elección de carrera influyen tanto factores motivacionales intrínsecos como extrínsecos, donde son igualmente fundamentales los atributos propios de la carrera (características preferidas).

\section{METODOLOGÍA}

La investigación tuvo un enfoque cuantitativo, de tipo descriptivo, alcance transeccional y de diseño no experimental, que buscó probar que las motivaciones y preferencias que determinan la decisión por estudiar ingeniería comercial, difieren entre estudiantes de los sistemas educativos público y privado.

El estudio se desarrolló en La Serena, ciudad del norte de Chile, capital económica de la Región de Coquimbo. Constituye parte de una conurbación con la ciudad colindante de Coquimbo, que totalizan una población estimada de más de 449 mil habitantes en 2017, estableciéndose como la cuarta área urbana más relevante del país (BCN, 2017). En La Serena existen tres universidades que imparten la carrera de ingeniería comercial: dos instituciones privadas (sedes), cuyas casas centrales se ubican en la ciudad de Santiago: $U$. Santo Tomás y la U. Central de Chile; y el único plantel público estatal de la Región de Coquimbo: la U. de La Serena. Cabe destacar que estas tres instituciones más la U. Católica del Norte, ubicada en la ciudad de Coquimbo, conforman el total de universidades que ofrecen la carrera en toda la Región de Coquimbo.

La muestra se constituyó de 106 estudiantes de primer año de la carrera de Ingeniería Comercial de instituciones de ambos sistemas educativos (público y privado): Universidad de La Serena (ULS), Universidad Santo Tomás (UST) sede La Serena y Universidad Central de Chile (UCEN) sede La Serena. El cálculo de la muestra se computó sobre una población total de 146 estudiantes, y utilizó un muestreo probabilístico estratificado con afijación proporcional (ULS: 52\%; UST: 27\% y UCEN: $21 \%$ ), con un nivel de significancia de $5 \%$, una probabilidad de $50 \%$ y un margen de error de $5 \%$.

El instrumento de medición utilizado correspondió a un cuestionario de preguntas cerradas, estructurado en dos secciones fundamentales, que fueron complementadas con siete preguntas de caracterización del encuestado (sexo, edad, educación escolar, con quien vive, lugar de origen, medio de pago y estudios universitarios previos) más una pregunta sobre el principal motivo por cursar la carrera, que contó con seis opciones de respuesta (vocación, crecimiento personal, buena salida laboral, costumbre familiar, estatus socioeconómico y habilidades académicas).

La primera sección tuvo por objeto medir los factores motivacionales del alumnado. Su contenido se basó en el Cuestionario de Metas de Elección de Carrera (CMEC) de García Ripa (2007), considerando los estudios previos realizados por Gámez y Marrero (2003). En total se formularon 43 preguntas bajo un formato de respuesta Likert de 5 puntos ( $1=$ Muy en desacuerdo, $2=$ En desacuerdo, $3=$ Indiferente, $4=$ De acuerdo y $5=$ Muy de acuerdo), las que fueron organizadas en nueves dimensiones de análisis: Afiliación e interés por las personas, Prestigio y poder, Innovación y emprendimiento, Sentimiento de logro personal, Vocación e interés por la carrera, Búsqueda de conocimiento, Valor social de la profesión, Motivación extrínseca y Posición económica. El detalle de las dimensiones y su descripción respectiva se aprecia en la tabla 1. La segunda sección del cuestionario tuvo por propósito determinar los atributos que mejor definen las preferencias por elegir la carrera de ingeniería comercial. Para su confección se realizó, inicialmente, una revisión de diferentes artículos especializados sobre el tema cuyos resultados se ajustaron a través de la aplicación de entrevistas a académicos expertos (3) y la opinión de estudiantes de Ingeniería Comercial (35 alumnos). Posteriormente, 
mediante un diseño ortogonal de datos se definieron 9 estímulos compuestos por 4 atributos y 10 niveles: acreditación de la carrera (Sí acreditada y No acreditada), reconocimiento institucional (universidad muy reconocida y universidad poco reconocida), enfoque del plan de estudios (emprendedor, social y administrativo) y arancel anual (USD 4.200, USD 4.500 y USD 4.800). Véase la figura 1. Los estudiantes debieron jerarquizar las tarjetas de la más a la menos preferida (1-9) usando la técnica de perfil total con presentación verbal, tal como lo sintetiza la tabla 2. Cabe señalar que la aplicación del cuestionario fue personal, autoaplicado, con participación voluntaria y anónima.

Para la examinación de las motivaciones se utilizaron pruebas inferenciales tradicionales, tales como: el Test de Shapiro Wilk para la verificación de la normalidad de los datos para muestras pequeñas, el test de Levene para la comprobación de la homogeneidad de varianzas, el test $T$ para la comprobación de medias cuando se cumplen los supuestos de normalidad y homogeneidad de varianzas, y el test no paramétrico de WilcoxonMann-Whitney cuando no. Además, se utilizó el test Chi-cuadrado para analizar la asociación de variables de naturaleza cualitativa.

Tabla1: Identificación de dimensiones, detalle descriptivo y cuantitativo

\begin{tabular}{|l|l|c|}
\hline Dimensiones motivacionales & Relacionado con & Ítems \\
\hline $\begin{array}{l}\text { Afiliación e interés por las } \\
\text { personas }\end{array}$ & $\begin{array}{l}\text { Afiliación, inclusión de los otros, interés por las personas, sensación de } \\
\text { pertenencia, responsabilidad social, equidad, solidaridad, pertenencia a } \\
\text { un grupo, vinculación. }\end{array}$ & 9 \\
\hline Prestigio y poder & Prestigio, poder sobre las personas. & 2 \\
\hline Innovación y emprendimiento & Interés por crear una empresa, resolver problemas sociales. & 2 \\
\hline Sentimiento de logro personal & $\begin{array}{l}\text { Superación de obstáculos, desarrollo de las potencialidades humanas, } \\
\text { de la identidad, éxito y satisfacción personal, adquisición de } \\
\text { recompensas, adquisición de diversión. }\end{array}$ & 6 \\
\hline Vocación e interés por la carrera & Vocación, ganas por estudiar y ejercer por la profesión. & 3 \\
\hline Búsqueda de conocimiento & $\begin{array}{l}\text { Importancia del tema, de la tarea, compromiso por el conocimiento, evitar } \\
\text { la ignorancia, ser experto, adquisición de nuevas habilidades, mejora. }\end{array}$ & 7 \\
\hline Valor social de la profesión & $\begin{array}{l}\text { Características sociales de la profesión, cómo es vista por la sociedad y } \\
\text { por los medios de comunicación. }\end{array}$ & 3 \\
\hline Motivación extrínseca & $\begin{array}{l}\text { Acciones o/y presiones del entorno familiar, del círculo de amistades, } \\
\text { grado de esfuerzo, evitar valoraciones negativas, obtención de notas, } \\
\text { recompensas y juicios positivos, influencias de la educación previa, de la } \\
\text { moda, aprobación de padres, profesores. }\end{array}$ & 9 \\
\hline Posición económica & Buena posición, sueldo reconocido, nivel económico superior. \\
\hline
\end{tabular}

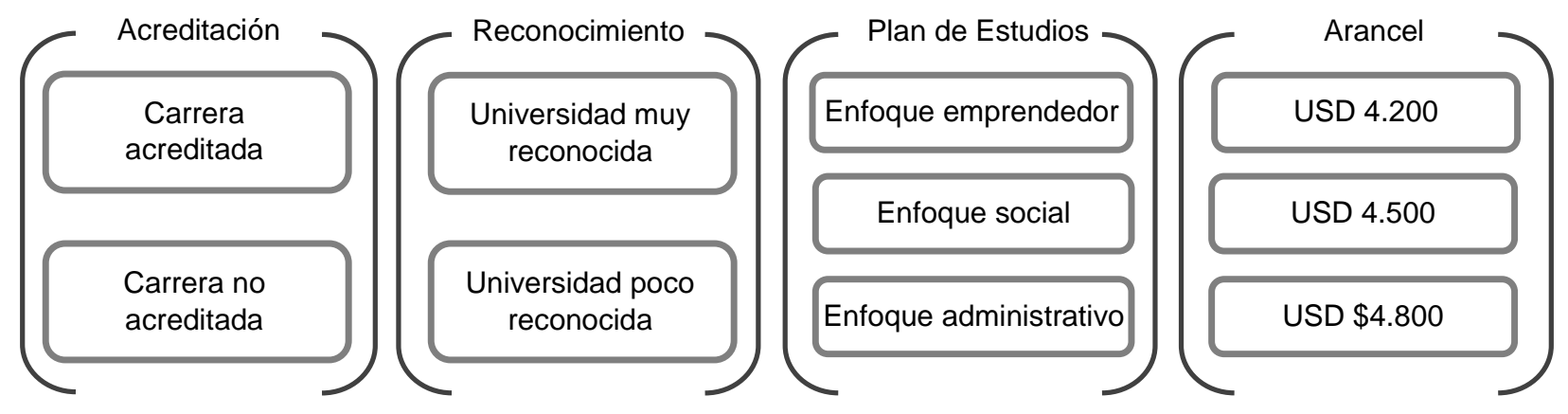

Fig. 1: Atributos y niveles del estudio

Tabla 2: Perfiles de las tarjetas generadas para la investigación

\begin{tabular}{|l|c|c|c|c|c|c|c|c|c|}
\hline Atributo & Tarjeta 1 & Tarjeta 2 & Tarjeta 3 & Tarjeta 4 & Tarjeta 5 & Tarjeta 6 & Tarjeta 7 & Tarjeta 8 & Tarjeta 9 \\
\hline Acreditación & No & Sí & Sí & Sí & No & Sí & Sí & Sí & No \\
\hline Arancel (USD) & 4.800 & 4.800 & 4.500 & 4.500 & 4.500 & 4.200 & 4.200 & 4.800 & 4.200 \\
\hline $\begin{array}{l}\text { Plan de } \\
\text { estudios }\end{array}$ & $\begin{array}{c}\text { Empren- } \\
\text { dedor }\end{array}$ & Social & Social & $\begin{array}{c}\text { Empren- } \\
\text { dedor }\end{array}$ & $\begin{array}{c}\text { Adminis- } \\
\text { trativo }\end{array}$ & $\begin{array}{c}\text { Adminis- } \\
\text { trativo }\end{array}$ & $\begin{array}{c}\text { Empren- } \\
\text { dedor }\end{array}$ & $\begin{array}{c}\text { Adminis- } \\
\text { trativo }\end{array}$ & Social \\
\hline Reconocimiento & Alto & Alto & Alto & Bajo & Alto & Alto & Alto & Bajo & Bajo \\
\hline
\end{tabular}


Por otra parte, para determinar la valoración de los atributos que explican las preferencias del alumnado se utilizó un modelo composicional multivariado (Análisis Conjunto), cuya fórmula conceptual fue como sigue:

$$
U_{t}=\alpha+\beta_{1} * x_{1 i}+\beta_{2} * x_{2 j}+\beta_{3} * x_{3 k}+\beta_{4} * x_{4 l}+e_{t}
$$

Donde, $U_{t}$ simboliza el orden de la tarjeta dado por el t-ésimo alumno, $x_{1 i}$ es el atributo acreditación de la carrera ( $i=1$-Sí y 2 -No), $x_{2 j}$ es el reconocimiento institucional $(j=1$-universidad muy reconocida y 2 -universidad poco reconocida), $x_{3 k}$ es el enfoque del plan de estudios ( $k=1$-enfoque emprendedor, 2-enfoque social y 3 enfoque administrativo), $x_{41}$ es el arancel anual (l=1-USD 4.200, 2-USD 4.500 y 3-USD 4.800), $\alpha$ es la constante de la regresión; $\beta_{1}, \beta_{2}, \beta_{3}$ y $\beta_{4}$ son los valores de utilidad parciales asociados a los niveles de cada atributo, y $\varepsilon_{t}$ es el error de estimación. Queda indicar que para el factor arancel se estableció una relación lineal inversa, acorde a la teoría económica, y los demás atributos se consideraron de naturaleza discreta.

Por último, la medición de la fiabilidad de la escala Likert para las motivaciones se obtuvo a través del cómputo del coeficiente alfa de Cronbach (0.81), mientras que para conocer la bondad de ajuste del modelo conjunto se usó el coeficiente Tau-b de Kendall (0.94), demostrando ambos indicadores que tanto el instrumento como el modelo propuesto cumplieron con las condiciones para ser aplicados.

\section{RESULTADOS Y DISCUSIÓN}

Se presentan los resultados de la investigación organizados en cuatro secciones: caracterización de la muestra, motivos de ingreso por la carrera, contraste de factores motivacionales y contraste de preferencias.

\section{Caracterización de la muestra}

La tabla 3 sintetiza las características sociodemográficas de la muestra. En ella se observa que en la universidad pública (ULS), la carrera de ingeniería comercial tiene una distribución equitativa entre hombres y mujeres ( $51 \%$ y $49 \%$ respectivamente), con estudiantes menores de 21 años $(78 \%)$, que provienen de las ciudades de La Serena (42\%), Ovalle (20\%) y Coquimbo (16\%), y que actualmente viven con sus padres $(53 \%)$ o arriendan un lugar para estudiar (26\%). Además, la mayor parte de los estudiantes de la institución provino de la educación escolar subvencionada (62\%) o pública (31\%), no ha cursado otras carreras previas (76\%) y declaró financiar sus estudios universitarios con gratuidad estatal (44\%) o apoyo de los padres (29\%).

Tabla 3: Aspectos sociodemográficos de la muestra

\begin{tabular}{|c|c|c|c|c|c|}
\hline \multirow[b]{2}{*}{ Dimensión } & \multirow[b]{2}{*}{ Ítem } & \multicolumn{2}{|c|}{ U. privada } & \multicolumn{2}{|c|}{ U. pública } \\
\hline & & Frecuencia & Participación & Frecuencia & Participación \\
\hline \multirow[t]{2}{*}{ Sexo } & Masculino & 22 & $43.1 \%$ & 27 & $49,1 \%$ \\
\hline & Femenino & 29 & $56.9 \%$ & 28 & $50,9 \%$ \\
\hline \multirow[t]{3}{*}{ Edad } & 18-20 años & 46 & $90.2 \%$ & 43 & $78,2 \%$ \\
\hline & 21-23 años & 2 & $3.9 \%$ & 10 & $18,2 \%$ \\
\hline & 24-26 años & 3 & $5.9 \%$ & 2 & $3,6 \%$ \\
\hline \multirow[t]{3}{*}{ Educación Escolar } & Privada & 10 & $19.6 \%$ & 4 & $7.3 \%$ \\
\hline & Subvencionada & 31 & $60.8 \%$ & 34 & $61.8 \%$ \\
\hline & Pública & 10 & $19.6 \%$ & 17 & $30.9 \%$ \\
\hline \multirow[t]{4}{*}{ Actualmente vive con } & Casa de sus padres & 39 & $76.4 \%$ & 29 & $52.7 \%$ \\
\hline & Pensión & 6 & $11.8 \%$ & 5 & $9.1 \%$ \\
\hline & Arrienda casa o depto. & 0 & $0.0 \%$ & 14 & $25.5 \%$ \\
\hline & Casa de familiar & 6 & $11.8 \%$ & 7 & $12.7 \%$ \\
\hline \multirow[t]{4}{*}{ Lugar de origen } & La Serena & 28 & $54.9 \%$ & 23 & $41.8 \%$ \\
\hline & Coquimbo & 10 & $19.6 \%$ & 9 & $16.4 \%$ \\
\hline & Ovalle & 2 & $3.9 \%$ & 11 & $20.0 \%$ \\
\hline & Otro & 11 & $21.6 \%$ & 12 & $21.8 \%$ \\
\hline \multirow[t]{3}{*}{ Forma de pago } & Beca & 3 & $5.9 \%$ & 8 & $14,5 \%$ \\
\hline & Crédito & 10 & $19.6 \%$ & 2 & $3,6 \%$ \\
\hline & Beca y crédito & 6 & $11.8 \%$ & 2 & $3.6 \%$ \\
\hline
\end{tabular}


Tabla 3: (continuación)

\begin{tabular}{|l|l|c|c|c|c|}
\hline \multirow{2}{*}{ Dimensión } & \multirow{2}{*}{ Ítem } & \multicolumn{2}{|c|}{ U. privada } & \multicolumn{2}{c|}{ U. pública } \\
\cline { 2 - 6 } & Frecuencia & Participación & Frecuencia & Participación \\
\hline \multirow{4}{*}{ Forma de pago } & Padres & 22 & $43.1 \%$ & 16 & $29.1 \%$ \\
\cline { 2 - 6 } & Personal & 1 & $2.0 \%$ & 2 & $3.6 \%$ \\
\cline { 2 - 6 } & Gratuidad & 0 & $0.0 \%$ & 24 & $43.6 \%$ \\
\cline { 2 - 6 } & Padres y crédito & 6 & $11.8 \%$ & 1 & $1.8 \%$ \\
\cline { 2 - 6 } & Padres y beca & 3 & $5.9 \%$ & 0 & $0.0 \%$ \\
\hline \multirow{2}{*}{$\begin{array}{l}\text { Estudios universitarios } \\
\text { previos }\end{array}$} & Sí & 11 & $21.6 \%$ & 13 & $23.6 \%$ \\
\cline { 2 - 6 } & No & 40 & $78.4 \%$ & 42 & $76.4 \%$ \\
\hline
\end{tabular}

En cambio, en las universidades privadas (UST y UCEN) se observa que la mayor parte del estudiantado es de sexo femenino (57\% mujeres y $43 \%$ hombres); casi la totalidad tiene menos de 21 años (90\%); provienen de las ciudades de La Serena (55\%) y Coquimbo (20\%); viven en la actualidad con sus padres (77\%); y financian sus estudios con el aporte de estos (43\%) o con crédito universitario (20\%). Además, en cuanto a la educación recibida, destaca que la mayoría proviene de establecimientos educacionales particulares subvencionados (61\%), y el resto, en igual distribución (20\% cada uno), de una educación privada y/o pública. Asimismo, no han estudiado otras carreras previamente (78\%).

\section{Motivos de ingreso por la carrera}

El análisis de los motivos por ingresar a estudiar Ingeniería Comercial según tipo de sistema (privado o público), mostró una asociación significativa entre las variables (test chi-cuadrado con $p=0.013$ ), que permiten establecer lo siguiente: los estudiantes de las casas de estudios privadas eligen la carrera principalmente por la vocación o afinidad que sienten por la profesión en vez de otra $(37.3 \%)$ y por la salida laboral (alta empleabilidad) que ofrece (25.5\%). Mientras que, los estudiantes pertenecientes a la universidad pública estatal de la comuna prefieren la carrera, principalmente, debido a su buena salida laboral (36.4\%) y por el crecimiento personal (34.5\%) que reporta lograr el perfil de egreso del programa (conocimientos, habilidades, actitudes y valores). Llama la atención la proporción de estudiantes del sistema privado que elige la carrera por costumbre familiar $(9.8 \%)$ en contraposición a los educandos del sistema público $(0 \%)$. Lo que supondría un rol más activo de la familia sobre su proceso decisional (factor social). Véase la figura 2.

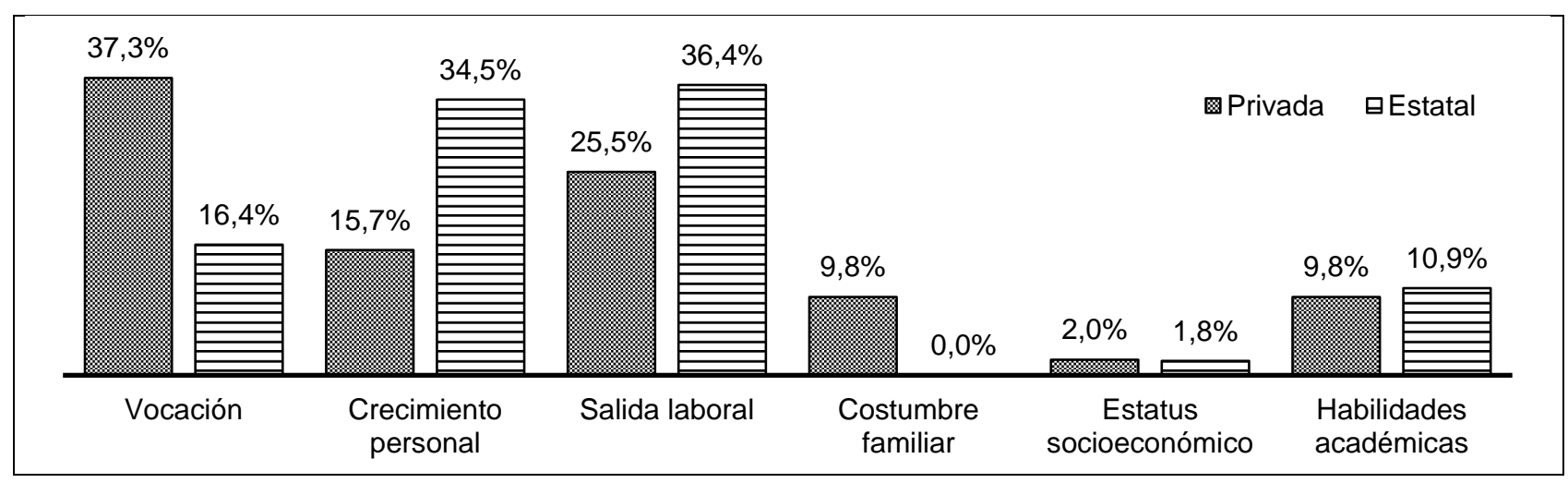

Fig. 2: Motivos por ingresar a estudiar Ingeniería Comercial

Es interesante denotar el trabajo de Ashari et al. (2019), en el cual mostró que el conocimiento de la carrera es un predictor significativo de su elección. Esto coincide con otros estudios que muestran que el conocimiento profesional, la conciencia laboral y el aprendizaje sobre el mundo laboral, influirían en la elección de carrera (Marinas et al., 2016; Nyamwange, 2016). Así, información referida a la profesión, el entorno laboral y salarios, facilitaría elegir estudiar ingeniería comercial, reconocida por su buena empleabilidad y altos ingresos.

\section{Contraste de factores motivacionales}

En cuanto a los factores motivacionales que influyen en la elección de la carrera de Ingeniería Comercial, casi la totalidad de los componentes (8/9), mostraron medias homogéneas, que no evidenciaron diferencias significativas entre estudiantes del régimen público y privado (Véase la tabla 4). Los resultados, ordenados de mayor a menor, fueron los siguientes: la innovación y emprendimiento (4.41), la vocación e interés por la carrera (4.36), el sentimiento de logro personal (4.35) y el valor social de la profesión (4.09). Le siguieron 
(media entre 3 y 4): la posición económica $(3,99)$, prestigio y poder (3.92), afiliación e interés por las personas $(3,54)$ y búsqueda de conocimiento $(3,39)$.

De lo anterior, es relevante y positivo acentuar, primero, el alto puntaje asociado al factor vocacional, considerado un determinante clave del progreso académico y permanencia en la universidad (Esteban et al., 2017), además de condición básica para ejercer íntegramente una profesión (Flores et al., 2019). Segundo, evidenciar que las motivaciones intrínsecas de los estudiantes de ambos sistemas se relacionan mayormente con la vocación y gusto por la carrera, y las motivaciones extrínsecas, a las buenas expectativas de empleabilidad e ingresos (Alonso-Dos-Santos et al., 2017). Y cuarto, confirmar que un atributo clave de elección de la carrera es su vinculación con el emprendimiento e innovación, es decir, la formación que brinda a sus estudiantes para emprender, ya sea mediante el desarrollo de iniciativas propias o para innovar dentro de organizaciones de terceros.

Tabla 4: Resumen de prueba de hipótesis para la comprobación de medias. * Prueba T de comparación de medias, ** Prueba U de Mann-Whitney

\begin{tabular}{|l|c|c|c|}
\hline \multirow{2}{*}{ Factor motivacional } & \multirow{2}{*}{ Universidad } & \multicolumn{2}{|c|}{ Resumen de prueba de hipótesis } \\
\cline { 3 - 4 } & Estadístico de prueba & Valor - $p$ \\
\hline Afiliación e interés por las personas & Privada / Pública & $-1.180^{*}$ & 0.241 \\
\hline Prestigio y poder & Privada / Pública & $0.731^{\star}$ & 0.466 \\
\hline Innovación y emprendimiento & Privada / Pública & $-1.022^{\star *}$ & 0.307 \\
\hline Sentimiento de logro personal & Privada / Pública & $-0.500^{* *}$ & 0.617 \\
\hline Vocación e interés por la carrera & Privada / Pública & $-0.201^{* *}$ & 0.841 \\
\hline Búsqueda de conocimiento & Privada / Pública & $-0.524^{*}$ & 0.601 \\
\hline Valor social de la profesión & Privada / Pública & $-0.208^{* *}$ & 0.836 \\
\hline Motivación extrínseca & Privada / Pública & $2.664^{*}$ & 0.009 \\
\hline Posición económica & Privada / Pública & $-0.073^{* *}$ & 0.941 \\
\hline
\end{tabular}

Por otro lado, y no obstante su bajo promedio (inferior a 3), la única dimensión que presentó diferencias significativas fue la de motivación extrínseca $(p=0.009)$, que resultó ser más valorada por los alumnos de establecimientos privados. El análisis de los reactivos de este componente, sintetizados en la tabla 5, mostró que las principales diferencias se perciben en que para los estudiantes del sistema privado es más fuerte la influencia de familiares y amigos (por ejemplo: "ingresé a estudiar esta carrera porque mis padres y/o amigos me lo han aconsejado"), la percepción sobre la factibilidad de cursar exitosamente la carrera (por ejemplo: "entré a estudiar esta carrera porque creo que me va a resultar fácil cursarla" o "esta carrera no es muy difícil para estudiar"), y, en menor medida por la imposibilidad de cursar otra carrera (por ejemplo: "no tenía la posibilidad de estudiar la carrera que verdaderamente deseaba").

Tabla 5: Prueba T de comparación de medias para las motivaciones extrínsecas por estudiar Ingeniería Comercial

\begin{tabular}{|l|c|c|c|c|}
\hline \multirow{2}{*}{ Motivación extrínseca } & \multicolumn{2}{|c|}{ Medias } & \multicolumn{2}{c|}{ Prueba de hipótesis } \\
\cline { 2 - 4 } & Privada & Pública & $\begin{array}{c}\text { Estadístico } \\
\text { de prueba }\end{array}$ & Valor - $p$ \\
\hline Ingresé a esta carrera para no tener que ponerme a trabajar & 1.71 & 1.87 & -0.881 & 0.380 \\
\hline $\begin{array}{l}\text { Entré a estudiar esta carrera porque me sentía presionado por mi } \\
\text { familia }\end{array}$ & 1.82 & 1.62 & 1.065 & 0.290 \\
\hline $\begin{array}{l}\text { Ingresé a estudiar esta carrera porque mis padres y/o amigos me lo } \\
\text { han aconsejado }\end{array}$ & 2.92 & 2.38 & 2.234 & 0.028 \\
\hline $\begin{array}{l}\text { Me considero una persona con problemas y a través de la carrera } \\
\text { dejaré de tenerlos }\end{array}$ & 2.35 & 2.33 & 0.114 & 0.910 \\
\hline $\begin{array}{l}\text { Entré a estudiar esta carrera porque creo que me va a resultar fácil } \\
\text { cursarla }\end{array}$ & 2.98 & 2.42 & 2.887 & 0.005 \\
\hline $\begin{array}{l}\text { No tenía la posibilidad de estudiar la carrera que verdaderamente } \\
\text { deseaba }\end{array}$ & 2.37 & 1.89 & 1.755 & 0.082 \\
\hline Es la opción que tuve para irme a estudiar a otro lado & 2.35 & 2.18 & 0.754 & 0.453 \\
\hline Esta carrera no es muy difícil para estudiar & 3.10 & 2.40 & 3.266 & 0.001 \\
\hline No puedo quedarme en mi casa sin hacer nada & 3.65 & 3.51 & 0.581 & 0.563 \\
\hline
\end{tabular}


Lo anterior, además de confirmar la influencia de los familiares y cercanos en la decisión de carrera, descubiertos por otros estudios (Karabulut y Erkanli, 2017), abre espacio para profundizar el análisis de las causales que producen que las motivaciones extrínsecas sean más altas para los estudiantes de las universidades privadas, en especial, lo referido a los aspectos normativos y de control conductual percibido. Es decir, analizar en qué medida el estudiante está motivado para cumplir con las creencias de las personas que le rodean y en qué medida se siente con la capacidad para cometer con éxito las exigencias propias de la carrera. Asimismo, indagar si existen algunas de estas motivaciones que sean propias del estudiante de ingeniería comercial.

\section{Contraste de preferencias}

El modelo composicional multivariado permitió identificar los atributos preferidos por los estudiantes al elegir estudiar la carrera de ingeniería comercial. En la figura 3 se observa que las preferencias de los alumnos de ambos tipos de sistemas no presentan diferencias mayores; por lo tanto, ambos consideran como atributo más valorado, la Acreditación de la carrera (privado=35.1\% y público=32.7\%). El atributo Enfoque del plan de estudios fue el segundo más valorado (privado=27.7\% y público=27.5\%), donde el alumnado privilegió un Enfoque emprendedor, por sobre uno Administrativo o Social. Como tercer atributo valorado se registró el Reconocimiento institucional (privado $=20.5 \%$ y público $=24.7 \%$ ), esto quiere decir que mientras más reconocimiento o prestigio tenga el plantel universitario, más preferido será este por los estudiantes. Finalmente, el factor de menor importancia fue el Costo del arancel anual, prefiriendo el tanto los estudiantes del sistema privado (16.7\%) como público (15.1\%) costos más bajos (USD 4.200).

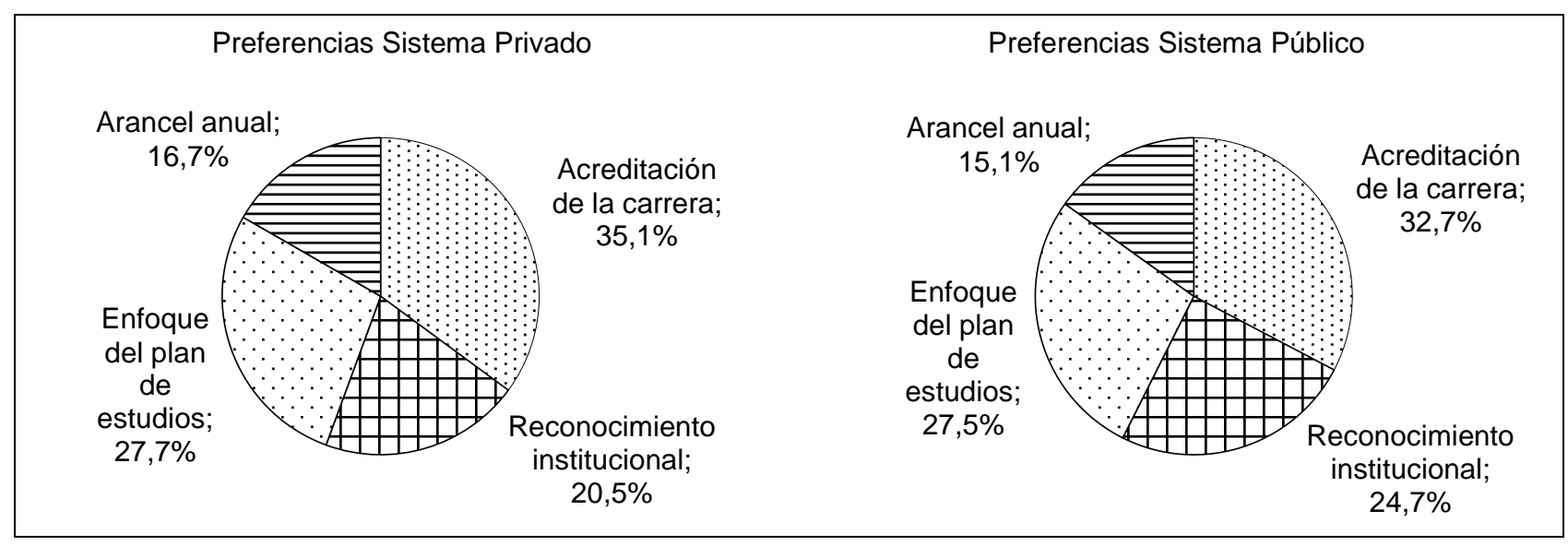

Fig. 3: Importancia relativa asociada a cada atributo según sistema educativo

Del contraste de los resultados previos se aprecia, primero, que los encuestados en general valoran la acreditación como un atributo clave que guía su decisión de estudiar ingeniería comercial (34\% promedio), lo que demuestra coincidencia con otras investigaciones (Araya-Pizarro et al., 2015; Maniu y Maniu, 2014). Segundo, los alumnos del sistema público otorgan una mayor importancia relativa al atributo reconocimiento institucional que los alumnos del sistema privado. Tercero, el enfoque del plan de estudios, representado por su programa curricular, demuestra ser un factor muy importante para el estudiantado de ambos sistemas (importancia relativa homogénea). Y cuarto, se evidencia que, para los estudiantes de la carrera de ingeniería comercial, la calidad es un factor decisivo que define sus preferencias, pues tanto la acreditación del programa como el prestigio de la universidad, son considerados indicadores representativos de la calidad educativa. Así cabría suponer que la información entregada por los sistemas de medición de la calidad en Chile y los rankings universitarios juegan un rol importante en la decisión de los jóvenes al momento de elegir la carrera e institución donde cursar sus estudios superiores.

En la figura 4 se aprecia, de acuerdo a las utilidades obtenidas, las características predilectas de Ingeniería Comercial escogidas por los estudiantes de los planteles tanto privados como público, donde destaca que la carrera debe estar acreditada (privada=1.5 y pública=1.4), ser impartida por una universidad muy reconocida (privada $=0.7$ y pública $=1.0$ ), el enfoque del programa de estudios debe ser emprendedor (privada=0.7 y pública $=0.5$ ) y tener un costo anual de no más de USD 4.200 (privada=-0.6 y pública $=-0.5$ ). En general, las preferencias demostraron ser homogéneas entre sistemas educativos, destacando la existencia de una menor predilección por un plan de estudios con enfoque social para el caso del estudiantado del sistema público.

Con relación a este último punto, resulta llamativo que sean precisamente los jóvenes que prefieren estudiar en una universidad pública estatal, espacio que enarbola conceptos como justicia social y sociedad más justa, sean quienes menos valoren un programa curricular con enfoque social (tercer lugar), incluso más bajo que 
la jerarquía otorgada por el alumnado del sistema privado (segundo lugar). Esto conlleva a cuestionarse sobre cuáles son los aspectos que condicionan su falta de predilección en su decisión de carrera.

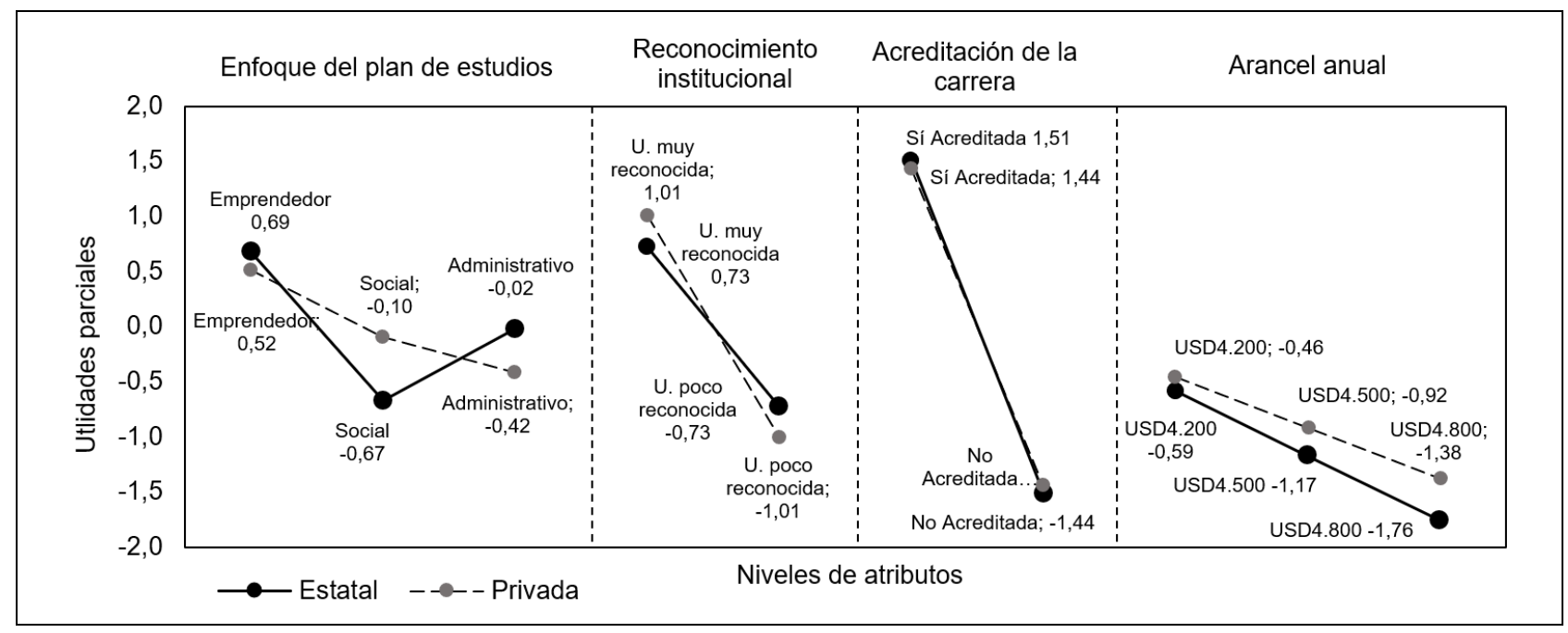

Fig. 4: Utilidades estimadas por el modelo según sistema educativo

\section{CONCLUSIONES}

De los resultados mostrados, sus análisis y posterior discusión, se obtienen las siguientes conclusiones: (1) Las motivaciones y preferencias que determinan la decisión por estudiar ingeniería comercial, no difieren mayormente entre estudiantes de los sistemas educativos público y privado. Las motivaciones del alumnado de ambos sistemas se relacionan, intrínsecamente, con la vocación y gusto por la carrera, y extrínsecamente a las expectativas de empleabilidad e ingresos. Ello evidencia que el conocimiento de la carrera es un predictor significativo de su preferencia (empleabilidad e ingresos); (2) La motivación extrínseca es más fuerte para los estudiantes del sistema privado, donde se observa una mayor influencia de cercanos (norma subjetiva) y de control conductual percibido (autoeficacia); (3) Los atributos determinantes de la elección de la carrera, para el estudiantado de ambos sistemas, se relacionan con la calidad (acreditación y reconocimiento institucional) y el enfoque del plan de estudios (en emprendimiento); (4) Los alumnos del sistema público valoran más el prestigio de la universidad y menos un plan de estudios con enfoque social, que los estudiantes del sistema privado; (5) Por último, el contexto específico del estudio, da pie para que próximas investigaciones, basadas en un mayor alcance geográfico, puedan confirmar y generalizar los hallazgos conseguidos.

\section{REFERENCIAS}

Alonso-Dos-Santos, M., Jelves-Jara, M.J. y Mardones-Valdebenito, P.J., Posicionamiento de la Carrera de Ingeniería Comercial en la Región del Biobío, Rev. Iberoam. Educ. Super, 8(21), 138-153 (2017)

Araya-Pizarro, S.C., Rojas, L.E. y Fernández, J.J., Preferencias por un Programa de Posgrado: Un Análisis de los Factores que Contribuyen a la Elección de los Posgrados en Administración por Profesionales de la Región de Coquimbo, Chile, Revista Universitaria Ruta, 17(1), 63-76 (2015)

Ashari, Z.H.M., Azman, N. y Rasul, M.S., Factors Predicting Career Choice among Malaysian Students in Skills-Based Training Institutions, https://doi.org/10.1007/s10775-018-9366-5, International Journal for Educational and Vocational Guidance, 19(1), 19-39 (2019)

BCN., Reporte Estadístico Comunal 2017 Coquimbo, Biblioteca del Congreso Nacional de Chile, Santiago de Chile (2017)

Bergmark, U., Lundström, S., Manderstedt, L. y Palo, A., Why become a teacher? Student Teachers' Perceptions of the Teaching Profession and Motives for Career Choice, https://doi.org/10.1080/02619768.2018.1448784, European Journal of Teacher Education, 41(3), 266-281 (2018)

Bravo, G. y Vergara, M.A., Factores que Determinan la Elección de Carrera Profesional: en Estudiantes de Undécimo Grado de Colegios Públicos y Privados de Barrancabermeja, Psicoespacios, 12(20), 35-48 (2018).

Bukuluki, P., Höjer, S. y Jansson, B., Motives and Career Choices Among Ugandan Social Work Students, https://doi.org/10.1080/13691457.2017.1399252, European Journal of Social Work, 22(4), 712-724 (2019)

Cheng, W., How Intrinsic and Extrinsic Motivations Function among College Student Samples in Both Taiwan and the U.S., https://doi.org/10.1080/01443410.2018.1510116, Educational Psychology, 39(4), 430-447 (2019)

Deci, E.L. y Ryan, R.M., The "What" and "Why" of Goal Pursuits: Human Needs and the Self-Determination of Behavior, https://doi.org/10.1207/s15327965pli1104_01, Psychological Inquiry, 11(4), 227-268 (2000) 
Diwan, V., Minj, C., Chhari, N. y De Costa, A., Indian Medical Students in Public and Private Sector Medical Schools: Are Motivations and Career Aspirations Different? - Studies from Madhya Pradesh, India, https://doi.org/10.1186/1472-692013-127, BMC Medical Education, 13(127), 1-6 (2013)

Esteban, M., Bernardo, A., Tuero, E., Cervero, A. y Casanova, J., Variables that Influence Academic Performance and University Persistence, https://doi.org/10.1016/j.ejeps.2017.07.003, European Journal Of Education And Psychology, 10(2), 75-81 (2017)

Flores, M., Góngora, J.J, López, M.V. y Eraña, I.E., Why Become a Doctor? The Motivation of Students to Choose Medicine as a Professional Career, https://doi.org/10.1016/j.edumed.2019.01.002, Educacion Medica, 1-4 (2019)

Gámez, E. y Marrero, H., Metas y Motivos en la Elección de la Carrera Universitaria: Un Estudio Comparativo entre Psicología, Derecho y Biología, Anales de Psicología, 19(1), 121-131 (2003)

García-Ripa, M.I., Sánchez-García, M.I. y Risquez, A., Perfiles Motivacionales de Elección de Estudios en Estudiantes Universitarios de Nuevo Ingreso, https://doi.org/10.11144/javeriana.upsy17-3.pmee, Universitas Psychologica, 17(3), 1$11(2018)$

Ho, K.K.W., Lo, P. y otros seis autores, Intrinsic vs. Extrinsic Motivations of Master of Library and Information Science Students: A Cross-Cultural Comparative Study, https://doi.org/10.1177/0961000616664564, Journal of Librarianship and Information Science, 50(2), 141-156 (2018)

Karabulut, E. y Erkanli, H., Career Choices of Business Administration Students: A Turkish Case, https://doi.org/10.20491/isarder.2017.283, Journal of Business Research-Turk, 9(3), 1-15 (2017)

Maniu, I. y Maniu G., Educational Marketing: Factors Influencing the Selection of a University, Practical Application of Science, 2(3), 37-42 (2015)

Marinas, L.E., Igret, R.S., Marinas, C.V. y Prioteasa, E., Factors Influencing Career Choice: the Romanian Business and Administration Students' Experience, https://doi.org/10.14207/ejsd.2016.v5n3p267, European Journal of Sustainable Development, 5(3), 267-278 (2016)

Ng, E.S.W., Gossett, C.W., Chinyoka, S. y Obasi, I., Public vs Private Sector Employment: An Exploratory Study of Career Choice among Graduate Management Students in Botswana, https://doi.org/10.1108/PR-10-2014-0241, Personnel Review, 45(6), 1367-1385 (2016)

Nyamwange, J., Influence of Student's Interest on Career Choice Among First Year University Students in Public and Private Universities in Kisii County, Kenya, Journal of Education and Practice, 7(4), 96-102 (2016)

Ortiz, Y., López D. y Rivero O., Estudiantes Universitarios con Bajo Rendimiento Académico ¿Qué Hacer?, Edumecentro, 6(2), 272-278 (2014)

Quattrocchi, P., Flores, C. y otros seis autores, Motivación y Género en la Elección de Carrera, Revista de Educación y Desarrollo, 41, 27-35 (2017)

Rodriguez, A.B., Espinoza, J., Ramirez, L.J. y Ganga, A., Deserción Universitaria: Nuevo Análisis Metodológico, https://doi.org/10.4067/S0718-50062018000600107, Formación universitaria, 11(6), 107-118 (2018)

Rodríguez-Muñiz, L.J., Areces, D., Suárez-Álvarez, J., Cueli, M., y Muñiz, J., ¿Qué Motivos tienen los Estudiantes de Bachillerato para Elegir una Carrera Universitaria?, https://doi.org/10.23923/rpye2019.01.167, Revista de Psicología y Educación, 13(3), 1-15. (2018)

Said-Hung, E., Gratacós, G. y Valencia, J., Factores que Influyen en la Elección de las Carreras de Pedagogía en Colombia, https://doi.org/10.1590/S1517-9702201701160978, Educação e Pesquisa, 43(1), 31-48 (2017)

Sheldon, K.M. y Corcoran, M., Comparing the Current and Long-Term Career Motivations of Artists and Business-People: Is Everyone Intrinsic in the End?, https://doi.org/10.1007/s11031-018-9723-1, Motivation and Emotion, 43(2), 218-231 (2019)

Stover, J.B., Bruno, F.E., Uriel, F.E. y Fernández, M., Teoría de la Autodeterminación: Una Revisión Teórica, Perspectivas en Psicología: Revista de Psicología y Ciencias Afines, 14(2), 105-115 (2017)

Villamizar, G. y Delgado, J.A., Identificación de los Motivos para Estudiar Psicología en Estudiantes de Primer Año de la Corporación Universitaria de Investigación y Desarrollo -UDI, Revista Espacios, 38(30), 27 (2017)

Zuniga-Jara, S., Soria-Barreto, K. y Araya-Pizarro, S., Factores Determinantes en la Elección de una Universidad por Estudiantes de Ingeniería Comercial en la Región de Coquimbo, Chile, https://doi.org/10.4067/S071850062019000500061, Formación Universitaria, 12(5), 61-68 (2019) 